\title{
An allometric approach to quantify the extinction vulnerability of birds and mammals
}

\author{
J. P. Hilbers, ${ }^{1,6}$ A. M. Schipper, ${ }^{1}$ A. J. Hendriks, ${ }^{1}$ F. Verones,${ }^{1,2}$ H. M. Pereira,,${ }^{3,45}$ And M. A. J. Huijbregts ${ }^{1}$ \\ ${ }^{1}$ Department of Environmental Science, Institute for Wetland and Water Research, Faculty of Science, Radboud University \\ Nijmegen, PO Box 9010, NL-6500 GL, Nijmegen, The Netherlands \\ ${ }^{2}$ Industrial Ecology Programme, Department of Energy and Process Engineering, Norwegian University of Science and Technology \\ (NTNU), 7491, Trondheim, Norway \\ ${ }^{3}$ German Centre for Integrative Biodiversity Research (iDiv), Halle-Jena-Leipzig, Deutscher Platz 5e, 04103, Leipzig, Germany \\ ${ }^{4}$ Institute of Biology, Martin Luther University Halle-Wittenberg, Am Kirchtor 1, 06108, Halle (Saale), Germany \\ ${ }^{5}$ CIBIO/InBIO, Centro de Investigação em Biodiversidade e Recursos Genéticos, Cátedra Infraestruturas de Portugal \\ Biodiversidade, Universidade do Porto, Campus Agrário de Vairão, 4485-661, Vairão, Portugal
}

\begin{abstract}
Methods to quantify the vulnerability of species to extinction are typically limited by the availability of species-specific input data pertaining to life-history characteristics and population dynamics. This lack of data hampers global biodiversity assessments and conservation planning. Here, we developed a new framework that systematically quantifies extinction risk based on allometric relationships between various wildlife demographic parameters and body size. These allometric relationships have a solid theoretical and ecological foundation. Extinction risk indicators included are (1) the probability of extinction, (2) the mean time to extinction, and (3) the critical patch size. We applied our framework to assess the global extinction vulnerability of terrestrial carnivorous and non-carnivorous birds and mammals. Irrespective of the indicator used, large-bodied species were found to be more vulnerable to extinction than their smaller counterparts. The patterns with body size were confirmed for all species groups by a comparison with IUCN data on the proportion of extant threatened species: the models correctly predicted a multimodal distribution with body size for carnivorous birds and a monotonic distribution for mammals and non-carnivorous birds. Carnivorous mammals were found to have higher extinction risks than non-carnivores, while birds were more prone to extinction than mammals. These results are explained by the allometric relationships, predicting the vulnerable species groups to have lower intrinsic population growth rates, smaller population sizes, lower carrying capacities, or larger dispersal distances, which, in turn, increase the importance of losses due to environmental stochastic effects and dispersal activities. Our study is the first to integrate population viability analysis and allometry into a novel, process-based framework that is able to quantify extinction risk of a large number of species without requiring data-intensive, species-specific information. The framework facilitates the estimation of extinction vulnerabilities of data-deficient species. It may be applied to forecast extinction vulnerability in response to a changing environment, by incorporating quantitative relationships between wildlife demographic parameters and environmental drivers like habitat alteration, climate change, or hunting.
\end{abstract}

Key words: allometric relationships; critical patch size; extinction risk; mean time to extinction; population viability analysis; probability of extinction.

\section{INTRODUCTION}

A key goal in conservation biology is the protection of species to ensure stable, viable, and sustainable populations and ecosystems (Iwasa et al. 2000). To conserve biodiversity more effectively, it is important to predict the vulnerability of species to extinction (Purvis et al. 2000, Fujiwara 2007). Population viability analysis (PVA) is used to identify and evaluate threats to species and assess species' extinction risks and threat status

Manuscript received 23 October 2014; revised 17 August 2015; accepted 14 September 2015. Corresponding Editor: D. R. Strong.

${ }^{6}$ E-mail: j.hilbers@science.ru.nl
(Akçakaya and Sjögren-Gulve 2000). Within PVAs, various indicators are used to express the sensitivity of species to environmental change (Pe'Er et al. 2013), which can be subdivided into four main groups: (1) probability indicators, such as the probability of (quasi-) extinction, and probability of decline, (2) time indicators, such as the time to (quasi-)extinction, (3) area indicators, such as the minimum area required or critical patch size, and (4) population size indicators, such as the minimum viable population. The applicability of PVA is, however, typically limited by the availability of species-specific input data, such as the intrinsic population growth rate and maximum population density (Akçakaya and Sjögren-Gulve 2000). Due to data limitations, PVAs have mainly been restricted to local or 
regional settings and a limited number of species (Akçakaya and Sjögren-Gulve 2000). This constrains the opportunity to draw general conclusions regarding the relationships between extinction risks and species traits and hampers the general applicability of results in the global conservation of species (Gaston and Blackburn 1996, Kitzes and Merenlender 2013).

Typical wildlife demographic parameters required to calculate extinction risk indicators in PVAs, including the intrinsic population growth rate $\left(r_{\mathrm{m}}\right)$, carrying capacity $(K)$, and dispersal capacity $(d)$, have been related to body size (Pereira et al. 2004, Savage et al. 2004a, Hendriks 2007, Santini et al. 2013). These allometric relationships are well established and have a solid theoretical and ecological foundation (Brown et al. 2004). Yet, allometric relationships have seldom been incorporated into PVAs (but see, e.g., Kitzes and Merenlender 2013). This is remarkable, as allometric relationships are often based on data covering a large number of species from multiple taxonomic groups and spanning several orders of magnitude in body size (see, e.g., West and Brown 2005, Hendriks 2007).

Several studies found that extinction risk significantly correlates with body size (Gaston and Blackburn 1996, McKinney 1997, Cardillo et al. 2005). There are, however, large discrepancies between studies regarding the direction and strength of the correlation, which may relate to differences in taxonomic groups, habitats and spatial scale (McKinney 1997). For instance, Anderson et al. (2011) found no significant correlation between the national threat status and body size of more than 600 terrestrial mammals occurring in Canada, whereas Cardillo et al. (2005) found a significant positive association between global IUCN threat status and body size when investigating nearly 4000 species of non-marine mammals. Although increasing evidence from correlation studies underpins a positive association between body size and extinction risk, a sound analytical framework that explains the relationship between species vulnerability to extinction and body size is currently lacking (Gaston and Blackburn 2008, Smith and Lyons 2013).

The goal of this study was to develop an allometric framework that quantifies global extinction vulnerabilities using only limited data, facilitating the assessment of data-deficient species. Incorporating allometric relationships in extinction risk metrics enabled us to systematically investigate how extinction vulnerability changes in relation to body size. Three extinction indicators were included in the framework: (1) the probability of extinction (PE; Ginzburg et al. 1982), (2) the mean time to extinction (MTE; Foley 1994), and (3) the critical patch size (CPS; Skellam 1951, Pereira et al. 2004, Pereira and Daily 2006). These three indicators have been found to be suitable endpoints in conservation biology for identifying species that are most vulnerable to extinction (Pe'Er et al. 2013). The framework was applied to carnivorous and non-carnivorous birds and mammals as these species groups enjoy arguably the greatest public interest.

\section{Methods \\ Models}

Probability of extinction ( $P E$ ). - The probability of extinction is a commonly used indicator to predict the vulnerability of species to extinction (Pe'Er et al. 2013). The probability of extinction is defined as the probability that a population falls below a critical level after which extinction is imminent due to genetic and demographic stochastic effects (Ginzburg et al. 1982). In order to derive probabilities of extinction, we followed the model of Ginzburg et al. (1982):

$$
\mathrm{PE}=\left(\frac{N_{c} \cdot\left(1-\frac{N_{0}}{K}\right)}{N_{0} \cdot\left(1-\frac{N_{c}}{K}\right)}\right)^{2 r_{m} / \sigma_{r}^{2}}
$$

where PE is the probability of extinction (dimensionless between 0 and 1$), N_{c}$ is the critical population size below which extinction is imminent (in number of individuals), $N_{0}$ is the initial population size (in number of individuals), $K$ is the carrying capacity (in number of individuals), $r_{\mathrm{m}}$ is the intrinsic population growth rate (per unit of time), and $\sigma_{r}^{2}$ represents the variance in the intrinsic population growth rate $r_{\mathrm{m}}$, thus reflecting the influence of environmental stochasticity on $r_{\mathrm{m}}$.

Mean time to extinction (MTE).- The mean time to extinction is a measure that is obtained by recording the time until a modeled population, starting at a certain initial population size, reaches a threshold level of 1 individual or less, and taking the average of these times over multiple model simulations. To calculate mean time to extinctions, we used the numerical solution estimate of Foley (1994):

$\mathrm{MTE}=\frac{\sigma_{r}^{2}}{2 r_{m}^{2}}\left[e^{2 r_{m} \ln K / \sigma_{r}^{2}}\left(1-e^{-2 r_{m} \ln N_{0} / \sigma_{r}^{2}}\right)-\frac{2 r_{m} \ln N_{0}}{\sigma_{r}^{2}}\right]$

where MTE is the mean time to extinction (in number of years) and all other variables are the same as in the probability of extinction model of Ginzburg et al. (1982).

Critical patch size (CPS).- The critical patch size concept presumes that extinction is in essence a deterministic event primarily driven by habitat area. Due to edge effects, the loss of individuals via dispersal into non-suitable habitat increases when habitat area decreases. Extinction occurs when the habitat area is so small that the intrinsic population growth rate is not able to compensate for dispersal losses (Skellam 1951). This means that there is a minimum area of native habitat, the critical patch size (CPS), below which a population will decline to extinction due to individuals dispersing into non-suitable habitat (Pereira et al. 2004). To determine critical patch sizes, we 
followed the model of Pereira et al. (2004) and Pereira and Daily (2006):

$$
i \frac{I_{0}\left(i \sqrt{r_{1} /\left(2 \sigma_{d}^{2}\right)} L^{*}\right)}{I_{1}\left(i \sqrt{r_{1} /\left(2 \sigma_{d}^{2}\right)} L^{*}\right)}=\frac{1-\alpha}{\alpha} \sqrt{-\frac{r_{1}}{r_{2}}} \frac{K_{0}\left(\sqrt{-r_{2} /\left(2 \sigma_{d}^{2}\right)} L^{*}\right)}{K_{1}\left(\sqrt{-r_{2} /\left(2 \sigma_{d}^{2}\right)} L^{*}\right)}
$$

where $\alpha$ is the probability of a species moving outside of a native habitat patch at the habitat border (dimensionless), $r_{1}$ is the intrinsic population growth rate inside the native habitat patch, which is equal to $r_{\mathrm{m}}$ (per unit of time), $r_{2}$ is the intrinsic population growth rate outside the native habitat patch (per unit of time), $\sigma_{d}^{2}$ is the dispersal variance $\left(\mathrm{km}^{2}\right.$ per unit of time), $L$ is the diameter of the circular patch of native habitat $(\mathrm{km}), I_{n}$ and $K_{n}$ are Bessel functions of the first and second kind of order $n$, respectively, and $i$ represents the imaginary unit.

Numerically solving this equation to find the critical patch size results in:

$$
r_{m}=\frac{\ln \left(R_{0}\right)}{\tau_{g}}
$$

In turn, the generation time can be calculated based on body size combined with a production factor, which represents the average amount of energy directed to new biomass, and a body temperature correction factor, which is derived from biochemical reaction kinetics (Gillooly et al. 2001, Hendriks 2007):

$$
\tau_{g}=\frac{1}{q_{t} \cdot \gamma_{p} \cdot m^{-\kappa}}
$$

where $m$ represents the species body size (in $\mathrm{kg}$ ), $\kappa$ is the scaling exponent, $q_{\mathrm{t}}$ is a body temperature correction factor in comparison with the standard of $20^{\circ} \mathrm{C}$ (dimensionless), and $\gamma_{p}$ is the average production coefficient $\left(\mathrm{kg}^{\mathrm{K}} / \mathrm{d}\right)$. The intrinsic population growth rate can now be estimated via (Savage et al. 2004a,b, Hendriks 2007, Hendriks and Mulder 2012):

$$
\mathrm{CPS}=\pi \cdot\left[\min _{j_{0} \sqrt{2 \sigma_{d}^{2} / r_{1}} \leqslant L<\infty}\left\{\left(\frac{1}{(\alpha-1) \sqrt{r_{1}}} \frac{J_{0}\left(\sqrt{r_{1} /\left(2 \sigma_{d}^{2}\right)} L\right)}{J_{1}\left(\sqrt{r_{1} /\left(2 \sigma_{d}^{2}\right)} L\right)}+\frac{1}{\alpha \sqrt{-r_{2}}} \frac{K_{0}\left(\sqrt{r_{2} /\left(2 \sigma_{d}^{2}\right)} L\right)}{K_{1}\left(\sqrt{r_{2} /\left(2 \sigma_{d}^{2}\right)} L\right)}\right)^{2} / 2\right]^{2}\right.
$$

where $J_{n}$ is a Bessel function of the first kind of order $n$, and $j_{0}$ represent the smallest positive root of the Bessel function $J_{0}(x)$. To arrive at an extinction vulnerability indicator, we divided the CPS by the geographical range size $(A)$ of species, i.e., the total area within the outermost geographical limits of a species' occurrence range.

\section{Model parameterization}

The models were parameterized for four groups of terrestrial vertebrates: carnivorous birds, noncarnivorous (herbivorous and omnivorous) birds, carnivorous mammals, and non-carnivorous (herbivorous and omnivorous) mammals. Allometric relationships were used to estimate intrinsic population growth rate, current population density (needed to determine current population size), density-based carrying capacity (needed to determine carrying capacity), the variance in dispersal capacity and the variance in intrinsic population growth rate. Table 1 shows these allometric relationships including parameter values. To parameterize the geographical range size, the critical population size, and the probability of moving outside a patch, we resorted to empirical data and scientific literature.

Intrinsic population growth rate $\left(r_{m}, r_{1}\right.$, and $\left.r_{2}\right)$.- The intrinsic population growth rate can be estimated from the potential lifetime fecundity $\left(R_{0}\right.$, number of individuals) divided by the generation time $\left(\tau_{\mathrm{g}}\right.$, unit of time) (Hendriks and Mulder 2012):

$$
r_{m}=\ln \left(R_{0}\right) \cdot q_{t} \cdot \gamma_{p} \cdot m^{-\kappa}
$$

Regarding the intrinsic growth rates inside $\left(r_{1}\right)$ and outside $\left(r_{2}\right)$ the native habitat patch in the CPS calculations, we distinguished two groups of species based on habitat affinity, according to Pereira et al. (2004) and Pereira and Daily (2006): group A, species that use only native habitat; and group B, species that use both native and nonnative habitat. Species in group A are assumed to have an extremely high mortality rate in nonnative habitat $\left(r_{1}^{A}=r_{m}, r_{2}^{A}=-10\right)$, whereas species in group B are assumed to be unable to reproduce yet have natural mortality rates $(\mu)$ in nonnative habitat $\left(r_{1}^{B}=r_{m}, r_{2}^{B}=-\mu\right)$. Natural mortality rates were calculated from allometric relationships derived in Hendriks (2007); see Table 1.

Current population density $\left(N_{D}\right)$.- The current population density of species $\left(N_{\mathrm{D}}\right)$ can be estimated using body size as a sole predictor. Although, according to Damuth's law (Damuth 1981, 1987), the current population density follows a power-law relationship with body size with a scaling exponent of -0.75 independent of the taxonomic group or trophic level, steeper slopes have been found for carnivores (Carbone and Gittleman 2002, Jetz et al. 2004, Hendriks 2007, Isaac et al. 2011). This may be explained by an increase in territory size with increasing organism size due to an increase in territory overlap of carnivores (Hendriks 2007). Here, we set the scaling exponent at -0.75 for non-carnivores (Damuth 1981, 1987, Jetz et al. 2004, Hendriks 2007) and -0.88 for carnivores (Carbone and 


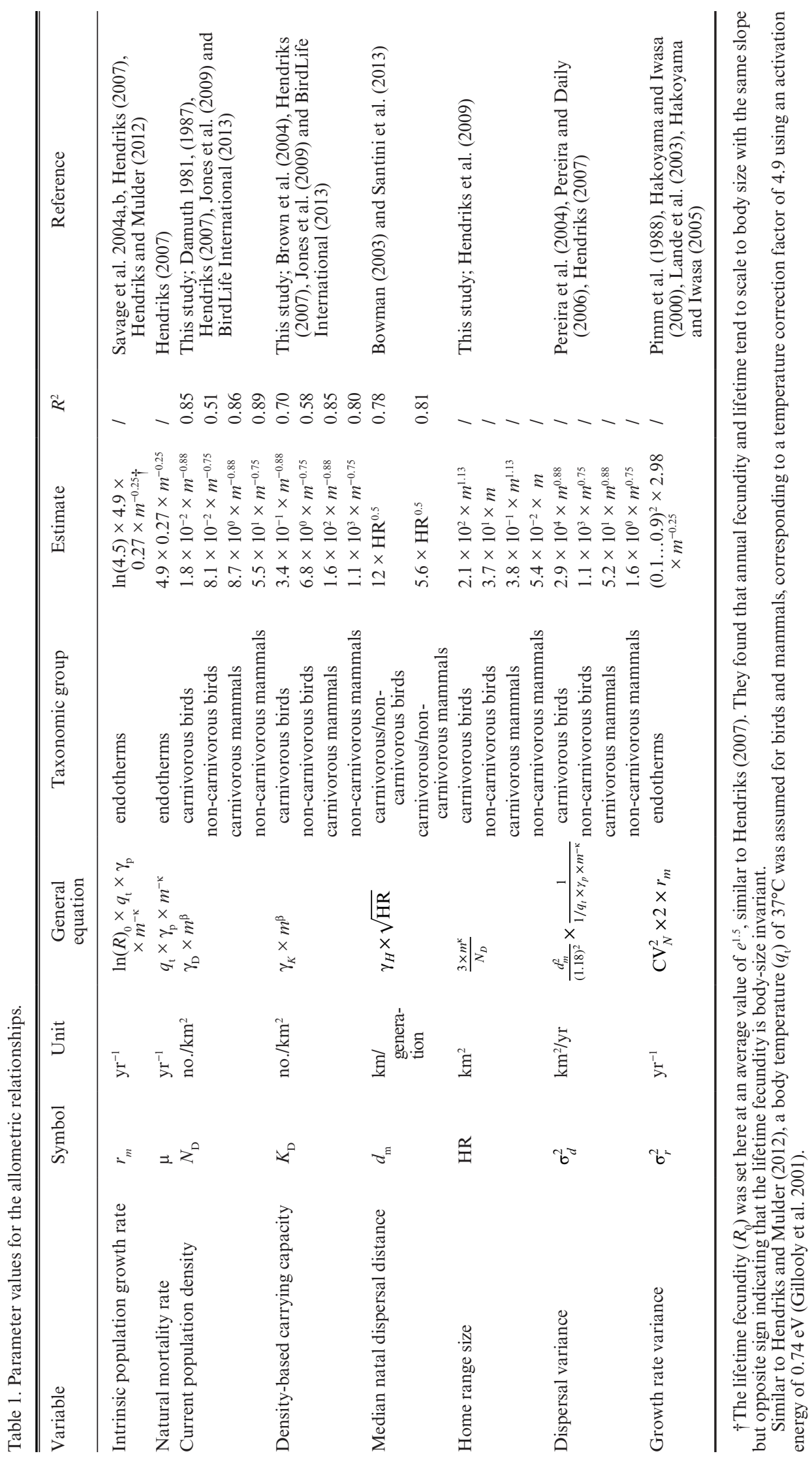


Gittleman 2002). Because we could not find allometric relationships for $N_{\mathrm{D}}$ for all four species groups, we used empirical data on the global current population densities of birds (618 non-carnivorous and 115 carnivorous species; BirdLife International 2013) and mammals (584 non-carnivorous and 78 carnivorous species; Jones et al. 2009) to derive corresponding intercept values. Because BirdLife International (2013) reports global population sizes instead of densities, we divided the population sizes by the reported geographical range sizes to arrive at population densities for birds. For each of the four species groups, we divided the observations into variably spaced logarithmic mass bins including 15 data points each. Per bin, a median population density was calculated (similar to, e.g., Savage et al. 2004b, Agosta and Bernardo 2013). The median densities were related to body size using Ordinary Least Squares (OLS) regression (see Table 1 and Appendix S1 in the Supplemental Material). To arrive at the current total number of individuals for a species $\left(N_{0}\right)$, we multiplied the current population densities $\left(N_{\mathrm{D}}\right)$ with the geographical range size $(A)$.

Carrying capacity $(K)$.-We defined the density-based carrying capacity $\left(K_{\mathrm{D}}\right)$ as the maximum population density of a species of a particular body size. Following the same approach as for the current population density, we set the slope for the carrying capacity at -0.75 for non-carnivores and at -0.88 for carnivores, and derived corresponding intercept values for all four species groups using OLS regression on the maximum densities reported for the mass bins, similar to the approach used for the current population density (Table 1, Appendix S1). To arrive at the maximum total number of individuals $(K)$, we multiplied the density-based carrying capacity by the geographical range size $(A)$.

Dispersal variance $\left(\sigma_{d}^{2}\right)$. - The annual dispersal variance $\left(\sigma_{d}^{2}\right)$ can be obtained from the median natal dispersal distance as follows (Pereira and Daily 2006):

$$
\sigma_{d}^{2}=\frac{d_{m}^{2}}{(1.18)^{2}} \cdot \frac{1}{1 / \mu}=\frac{d_{m}^{2}}{(1.18)^{2}} \cdot \frac{1}{1 /\left(q_{t} \cdot \gamma_{p} \cdot m^{-\kappa}\right)}
$$

where $d_{\mathrm{m}}$ represents the median natal dispersal distance $(\mathrm{km} /$ generation) and $1 / \mu$ reflects the mean life span (Hendriks 2007), which was needed to convert the dispersal variance from units of $\mathrm{km}^{2} /$ generation to units of $\mathrm{km}^{2} / \mathrm{yr}$.

We used allometric relationships of Bowman (2003), Hendriks et al. (2009) and Santini et al. (2013) to estimate the dispersal distance $d_{\mathrm{m}}$ (Table 1). It was shown that the median dispersal distance $(\mathrm{km} /$ generation) of both birds and mammals is proportionally related to the square root of home range size $\left(\mathrm{HR}\right.$, in $\left.\mathrm{km}^{2}\right)$ :

$$
d_{m}=\gamma_{H} \cdot \sqrt{\mathrm{HR}}
$$

where $\gamma_{H}$ is the home range scaling coefficient (generation $^{-1}$ ), which is equal to 12 for birds (Bowman 2003) and 5.6 for mammals (Santini et al. 2013). In turn, home range size (HR) has been related to body size by (Hendriks et al. 2009):

$$
\mathrm{HR}=\frac{3 \cdot m^{\mathrm{K}}}{N_{D}}
$$

in which the home range is calculated using the inverse of the current population density $\left(N_{\mathrm{D}}\right)$ and a correction factor for the number of individuals within a home range, which has been found to be $3 \times m^{\mathrm{K}}$ (Jetz et al. 2004). The median dispersal distance, and in turn the dispersal variance, is now solely dependent on body size.

Variance in intrinsic population growth rate $\left(\sigma_{r}^{2}\right)$.Both Ginzburg et al. (1982) and Foley (1994) assume that environmental stochasticity dwarfs demographic stochasticity, so that the variance in intrinsic population growth rate represents environmental stochasticity only. By assuming Stratanovich calculus in the stochastic fluctuations of the logistic population growth model, Hakoyama and Iwasa $(2000,2005)$ showed that squared coefficient of variation $(\mathrm{CV}$, dimensionless) of the size of a population equals the variance in intrinsic population growth rate divided by the intrinsic population growth rate of a species. The variance in intrinsic growth rate per unit of time can now be derived as follows:

$$
\sigma_{r}^{2}=2 r_{m} \cdot \frac{\operatorname{Var}[N]}{E[N]^{2}}=2 r_{m} \cdot \mathrm{CV}_{N}^{2}
$$

The coefficient of variation $\mathrm{CV}_{N}$ was found to be body size-independent (Sinclair 2003, Hendriks and Mulder 2012). From annual time series data of a wide variety of animal species, $\mathrm{CV}_{N}$ has been estimated to range between 10\% and 100\% (Pimm et al. 1988, Lande et al. 2003). In this study, we set $\mathrm{CV}$ to $0.1,0.5$, and 1.0 to represent weak, average, and strong fluctuations in population sizes.

Geographical range size (A).- In the literature, we did not find an unambiguous allometric relationship between the geographical range size and body size, as both triangular (Gaston and Blackburn 1996, Hendriks et al. 2009) and quadrangular (Agosta and Bernardo 2013) relationships have been reported. Therefore, we decided not to rely on an allometric relationship. Instead, we determined the 5th, 50th, and 95th percentiles of the range sizes within variably spaced logarithmic mass bins that included 50 data points each (see Appendix S2). To this end, we used empirical geographical range size data from IUCN (2014) of 6082 non-carnivorous birds, 1390 non-carnivorous mammals, 373 carnivorous birds, and 436 carnivorous mammals.

Critical population size $\left(N_{C}\right)$.-We set the critical population size at 500 individuals, because 500 individuals are generally thought of being sufficient for a population to avoid extinction due to genetic and demographic stochasticity in the long term. With this number of individuals the evolutionary potential is said to be maintained and the accumulation of deleterious genetic mutations to be avoided (Franklin 1980).

Probability of moving outside a patch $(\alpha)$. - To reflect variability in dispersal tendency, we used three values for 
the probability that an individual at the native habitat boundary will move outside its native habitat. Similar to Pereira et al. (2004) and Pereira and Daily (2006), we used $\alpha=0.5$ for species that are equally prone to stay within the habitat patch as to move outside it, $\alpha=0.1$ to cover species that avoid dispersing into non-native habitat and $\alpha=0.9$ for species that prefer dispersing away from their native habitat.

\section{Model application}

We calculated PE, MTE, and CPS for terrestrial carnivorous and non-carnivorous mammal and bird species by varying the body size from the smallest to the largest species for which geographical range size data was available (carnivorous birds, $0.041-11.3 \mathrm{~kg}$; non-carnivorous birds, 0.002-111 kg; carnivorous mammals, 0.002-162 kg; non-carnivorous mammals, $0.004-3825 \mathrm{~kg}$ ). Thereby, we have covered most of the body size ranges of all terrestrial bird and mammal species in the world.

\section{Model validation}

We compared the extinction vulnerabilities predicted by our framework with the proportion of extant threatened carnivorous and non-carnivorous birds and mammals. To this end, we collected data from IUCN (2014) on the Red List category and data from Jones et al. (2009) and Dunning (2007) on the body size of in total 371 carnivorous birds, 6069 non-carnivorous birds, 418 carnivorous mammals, and 1337 non-carnivorous mammals. For each of the four species groups, we divided the observations into 12 variably spaced logarithmic mass bins, including an equal number of data

a)

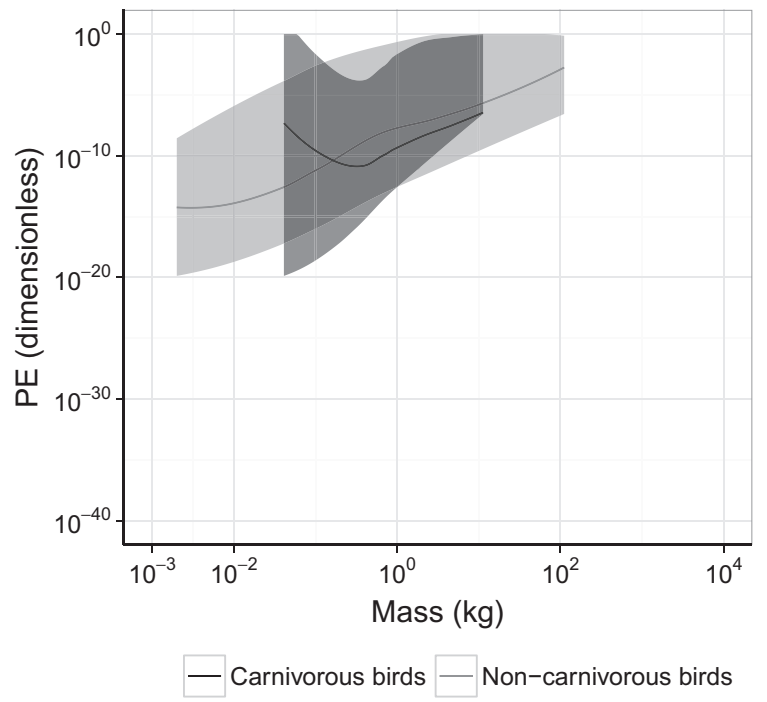

points each, and calculated the proportion of threatened species (i.e., IUCN categories "Vulnerable" to "Critically Endangered") per bin. Next, we performed Spearman's rank-order correlation analyses using the midpoints of the mass bins to compare the predictions of the three indicators with the relative frequency of currently threatened species as listed by IUCN. Finally, to test the robustness of the approach, we repeated this procedure using 6 and 24 variably spaced logarithmic mass bins, including an equal number of data points each, and mass bins including 50 data points each. All analyses were performed using the statistical software environment $\mathrm{R}$, version 3.0.1 ( $\mathrm{R}$ Core Team 2014), in which a locally weighted scatterplot smoothing (LOESS) was fit to the data.

\section{RESULTS}

We found a positive relationship between probability of extinction (PE) and body size (Fig. 1). For only carnivorous birds with small and median geographical range sizes was there no consistent positive relationship between PE and body size: carnivorous birds at intermediate body sizes had lower PE than their smaller and larger counterparts, i.e., the relationship between $\mathrm{PE}$ and body size was multimodal with smallest PE at intermediate body sizes (Fig. 1a). For mammals, PE tended to increase with body size irrespective of trophic habit or geographical range size (Fig. 1b). Further, birds had systematically higher PE than mammals and the $\mathrm{PE}$ of carnivorous mammals was in general higher than that of non-carnivorous mammals of the same size.

Mean time to extinction (MTE) was negatively related to body size (Fig. 2), i.e., the larger the body

b)

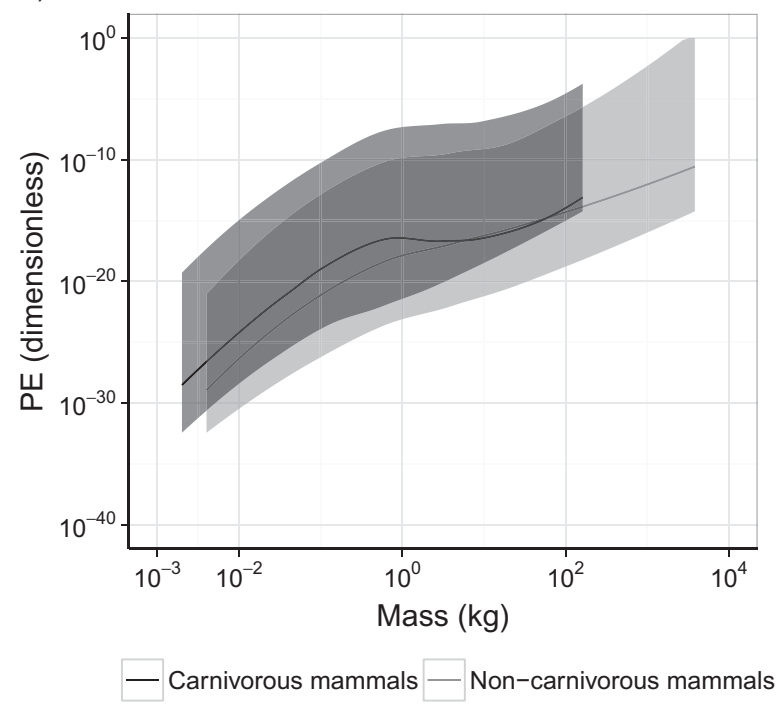

FIG. 1. Probability of extinction (PE; dimensionless) in relation to body size of (a) carnivorous and non-carnivorous birds and (b) carnivorous and non-carnivorous mammals for CV set at 0.5 . Lines represent the PE for species with median geographical range sizes and shaded areas represent the PE range for species with geographical range sizes between the 5th (upper border) and 95th percentiles (lower border). The results for PE with CV set at 0.1 and 1.0 can be found in Appendix S3. 
a)

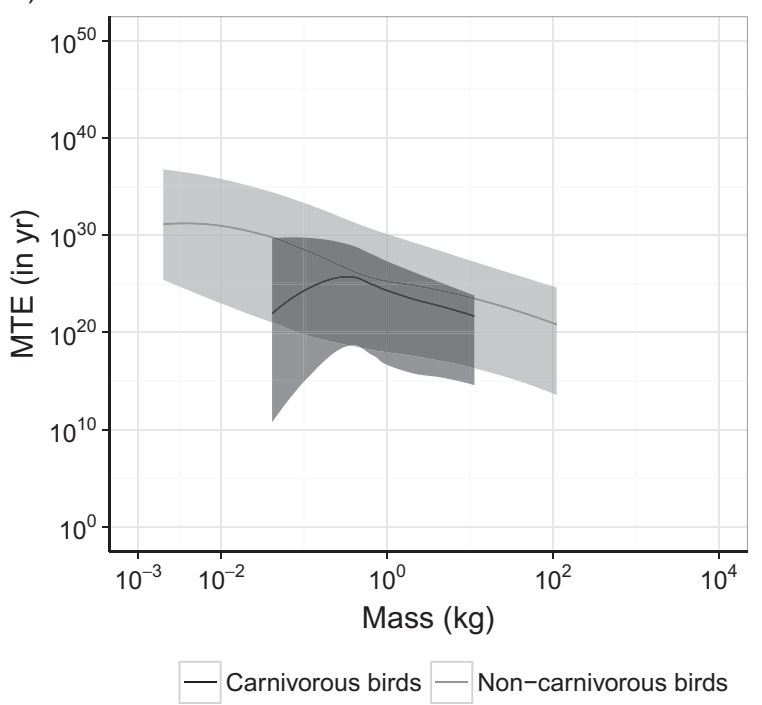

b)

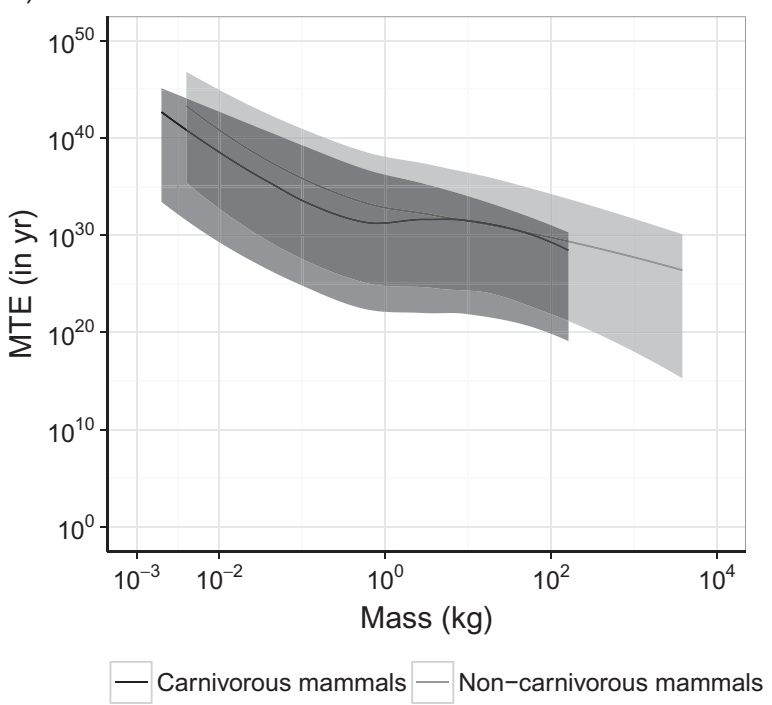

FIG. 2. Mean time to extinction (MTE; yr) in relation to body size of (a) carnivorous and non-carnivorous birds and (b) carnivorous and non-carnivorous mammals for CV set at 0.5. Lines represent the MTE for species with median geographical range sizes and shaded areas represent the MTE range for species with geographical range sizes between the 5th (lower border) and 95th percentiles (upper border). The results for MTE with CV set at 0.1 and 1.0 can be found in Appendix S3.

size, the lower the MTE. For only carnivorous bird species was there no consistent negative relationship between MTE and body size: carnivorous birds at intermediate body sizes had higher MTE than their smaller and larger counterparts. In addition, MTE was systematically lower for carnivores than noncarnivores, and birds had systematically lower MTE than mammals with similar geographical range sizes and body sizes.

Fig. 3a-d shows that the critical patch size was positively related with body size for both mammals and birds. Slopes ranged from 1.00 to 1.15 for noncarnivorous mammals/birds and 1.13 to 1.27 for carnivorous mammals/birds that use only native habitat (group A), and slopes were equal to 1.00 for noncarnivorous mammals/birds and to 1.13 for carnivorous mammals/birds that use both native and nonnative habitat (group B). The ratio of CPS to geographical range size $(A)$ was also positively related to body size, i.e., the larger the body size, the larger the ratio (Fig. 4). For only carnivorous bird species with small and median geographical range sizes was there no consistent positive relationship between $\mathrm{CPS} / A$ and body size: carnivorous birds at intermediate body sizes had lower CPS/ $A$ than their smaller and larger counterparts. Carnivores systematically showed a larger CPS and CPS/ $A$ than their non-carnivorous counterparts, and birds had a systematically larger CPS and CPS/ $A$ than mammals of the same trophic group and size. Comparing Figs. $3 \mathrm{a}, \mathrm{b}$ and $4 \mathrm{a}, \mathrm{b}$ with $3 \mathrm{c}$, $\mathrm{d}$ and $4 \mathrm{c}, \mathrm{d}$ indicates that species that use both native and nonnative habitat (group B) had smaller CPS and $\mathrm{CPS} / A$ compared to species that use only native habitat (group A).
The proportion of threatened species as listed by IUCN was positively related with body size (Fig. 5). For only carnivorous birds there was no consistent positive relationship between the proportion of threatened species and body size, as the proportion of threatened species was smallest at intermediate body sizes. These patterns were independent of the number of mass bins in which the observations were divided into (Appendix S4) and were comparable with the patterns found in all three modeled extinction indicators (Figs. 1, 2, 3 and 4). Correlation coefficients between our modelled extinction indicators and the proportion of threatened species as listed by IUCN ranged between 0.66 and 0.96 for species with median geographical range sizes ( $N=12$ bins, $P<0.001-0.05$; see Appendix S5 for the full results of the correlation analyses). In contrast to our model results, the IUCN data showed that mammals in general had a larger proportion of threatened species compared to birds, and carnivores and non-carnivores were similar in their proportion of threatened species.

\section{Discussion}

\section{Interpretation}

We combined PVA and allometric relationships to develop a framework that is able to quantify extinction vulnerability based on body size, and applied the framework to carnivorous and non-carnivorous birds and mammals. We found consistent results among the three indicators that we calculated. Large species were found to be more vulnerable to extinction than their smaller counterparts: larger species had higher probabilities of extinction, lower mean times to extinction, 
a)

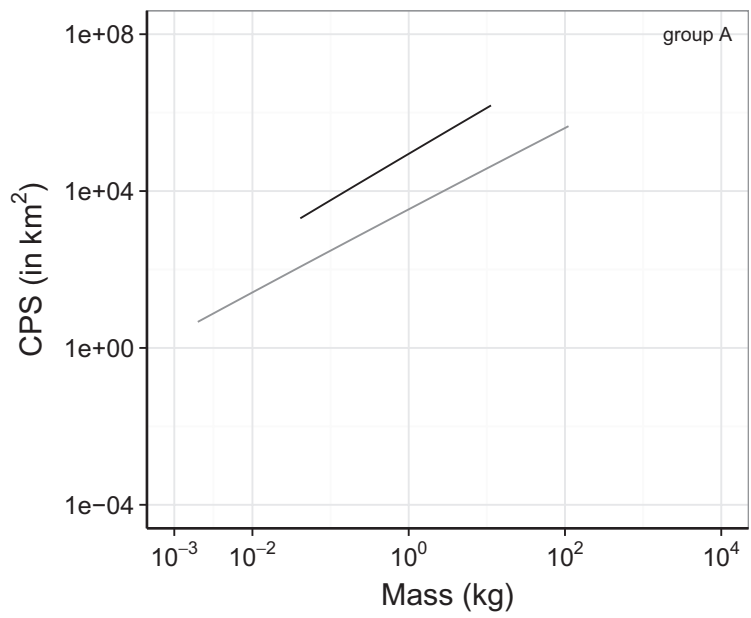

c)

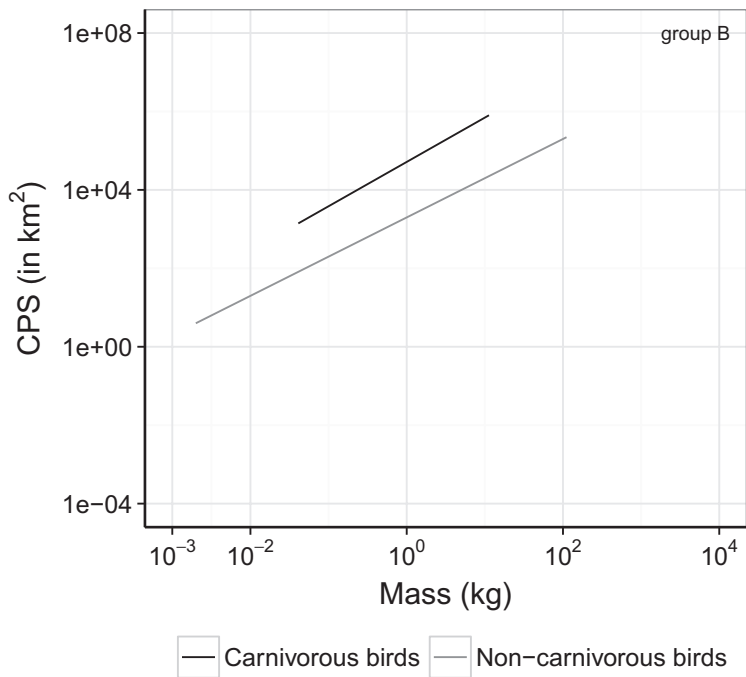

b)

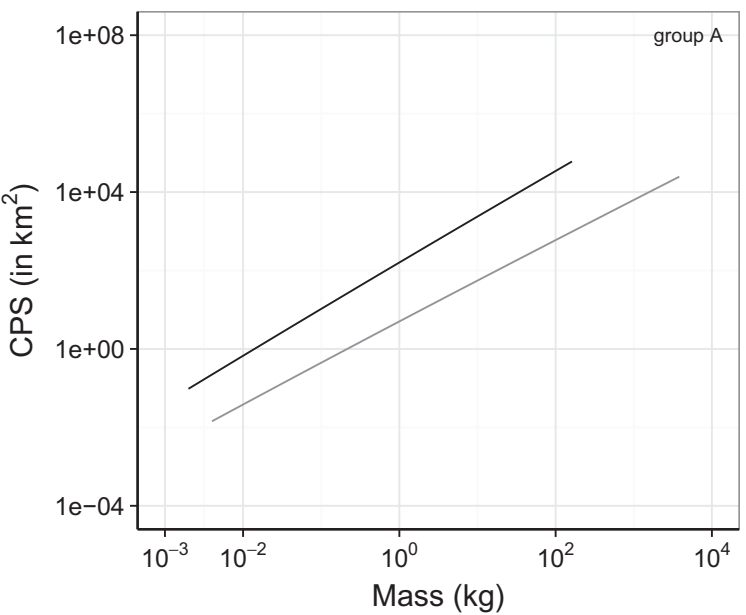

d)

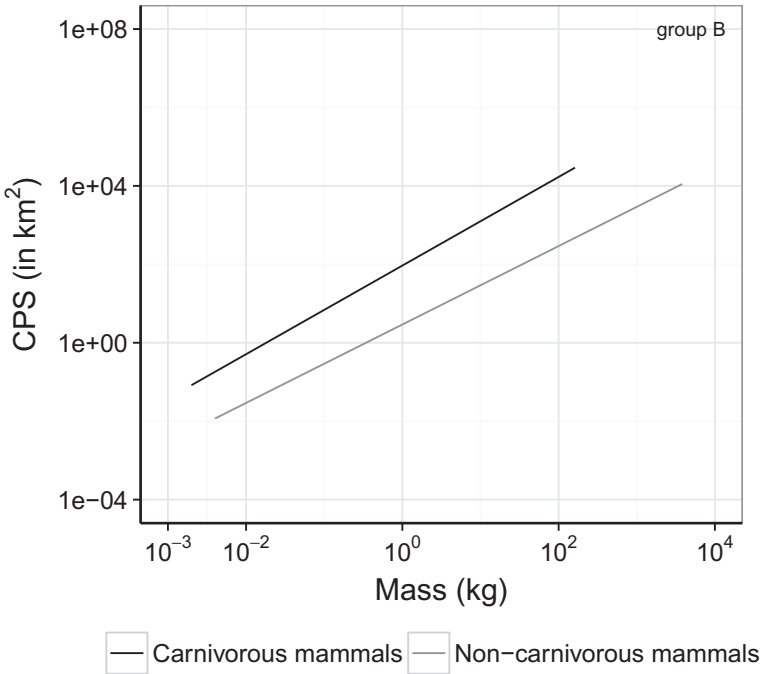

FIG. 3. Critical patch size (CPS; $\mathrm{km}^{2}$ ) in relation to body size for (a) carnivorous and non-carnivorous birds and (b) carnivorous and non-carnivorous mammals of habitat affinity group A, and (c) carnivorous and non-carnivorous mammals and (d) carnivorous and non-carnivorous birds of habitat affinity group B for $\alpha$ set at 0.5 . The results for CPS with $\alpha$ set at 0.1 and 0.9 can be found in Appendix S3.

and larger ratios of the critical patch size to the geographical range size. This can be explained by the allometric relationships predicting that species with a large body size have lower intrinsic population growth rates, smaller population sizes, lower carrying capacities, and larger dispersal variances, which, in turn, increase the importance of losses due to environmental stochastic effects and dispersal activities. These results are confirmed by findings of Pimm et al. (1988), McKinney (1997), Purvis et al. (2000), Cardillo et al. (2005), Davidson et al. (2009), Dirzo et al. (2014), and Pe'Er et al. (2014). One exception to this general pattern was carnivorous birds, which showed the lowest extinction vulnerability at intermediate body sizes $(0.2-6 \mathrm{~kg})$. Carnivorous bird species between 0.2 and $6 \mathrm{~kg}$ had relatively large geographical range sizes (Appendix S2) and, hence, large current population sizes and carrying capacities, which reduces the extinction proneness (Purvis et al. 2000). The multimodal pattern for carnivorous birds and the patterns of a monotonic nature for mammals and non-carnivorous birds in our extinction risk indicators were confirmed by the proportion of extant threatened species as listed by IUCN. In addition, studies on fossil records found a size selectivity in historical extinctions towards largerbodied species (Liow et al. 2008, Dirzo et al. 2014; but see Tomiya [2013] on extinctions at genus level).

In general, we found that birds were more vulnerable to extinction than mammals of the same size. The same was found for carnivorous birds and mammals compared to non-carnivorous birds and mammals, although differences were small. According to the allometric relationships used in this study, birds and carnivores have lower current population densities and density-based 
a)

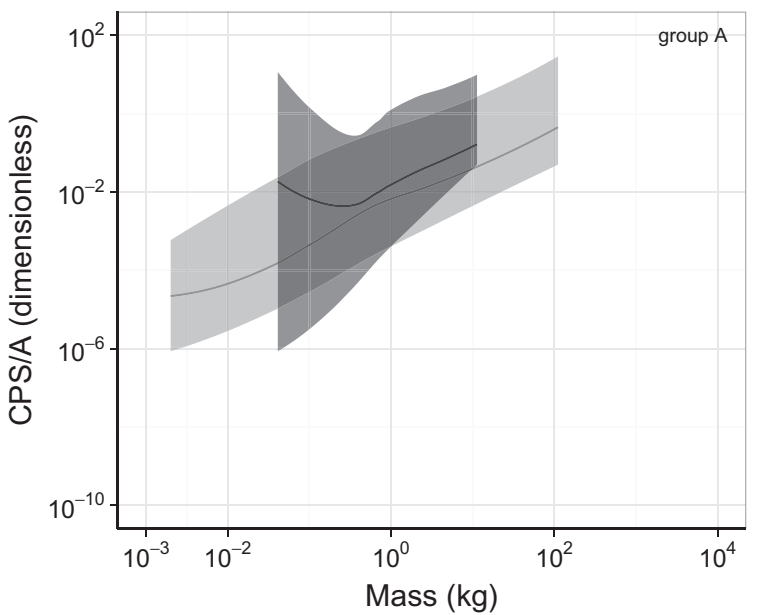

c)

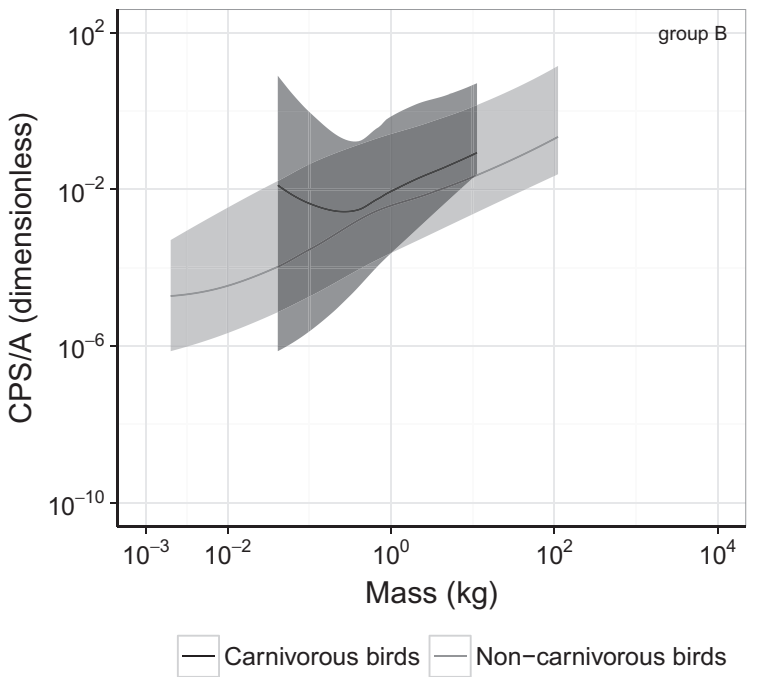

b)

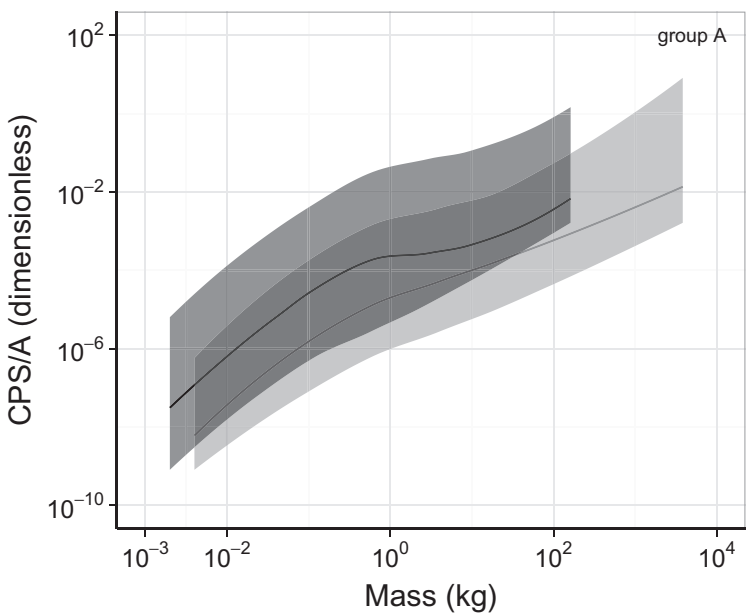

d)

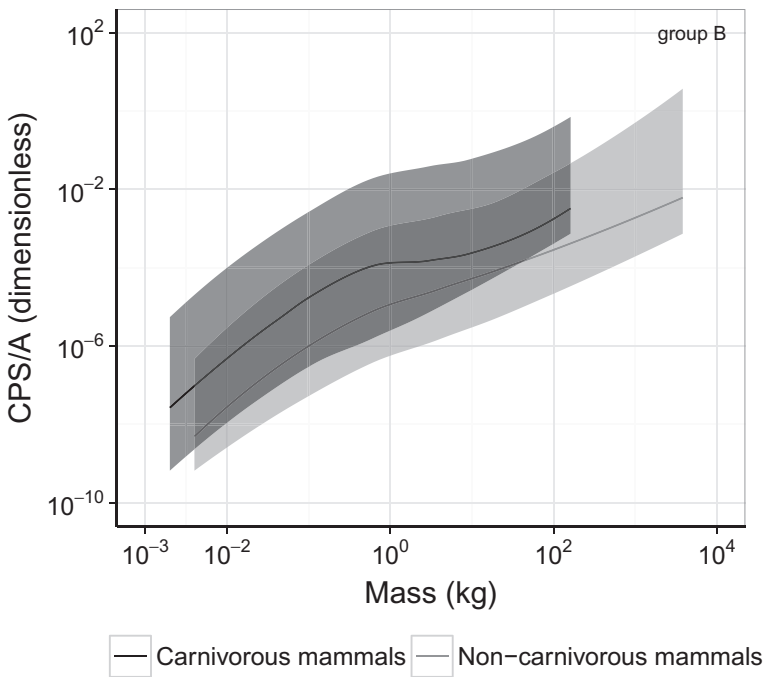

FIG. 4. The ratio of critical patch size to geographical range size (CPS/ $A$; dimensionless) in relation to body size for (a) carnivorous and non-carnivorous birds and (b) carnivorous and non-carnivorous mammals of group A dispersers, and (c) carnivorous and noncarnivorous mammals and (d) carnivorous and non-carnivorous birds of group B dispersers for $\alpha$ set at 0.5 . Lines represent CPS/ $A$ for species with median geographical range sizes and shaded areas represent the CPS/ $A$ range for species with geographical range sizes between the 5th (upper border) and 95th percentiles (lower border). The results for CPS/ $A$ with $\alpha$ set at 0.1 and 0.9 can be found in Appendix S3.

carrying capacities, yet similar intrinsic population growth rates and variances in growth rate (Table 1). Smaller population sizes and lower carrying capacities reduce the ability of species to withstand a series of bad years (Lande et al. 2003), so that environmental stochastic effects have a greater influence on birds and carnivores than on mammals and non-carnivores of the same size. Further, birds and carnivores tend to have larger median dispersal distances (Table 1), which result in large dispersal losses hence larger critical patch sizes. In accordance with these results, several studies have found that birds and carnivores are more prone to extinction than similar sized mammals and noncarnivores, respectively (McKinney 1997, Purvis et al. 2000, Pe'Er et al. 2014). However, IUCN Red List data showed that mammals are currently more threatened with extinction than birds, and that carnivores and non-carnivores have similar proportions of threatened species. These differences between IUCN data and our findings can be explained by the fact that our framework mainly captures intrinsic extinction vulnerability. Anthropogenic drivers of extinction, like habitat alteration, pollution and climate change, which might disproportionally affect certain species groups or body size ranges, are not yet included in the framework. Buchmann et al. (2013), for example, showed that mammals and larger species are more affected by habitat alteration than birds and smaller species, respectively.

Our results also show that the extinction vulnerabilities of bird and mammal species that have median or larger 
a)

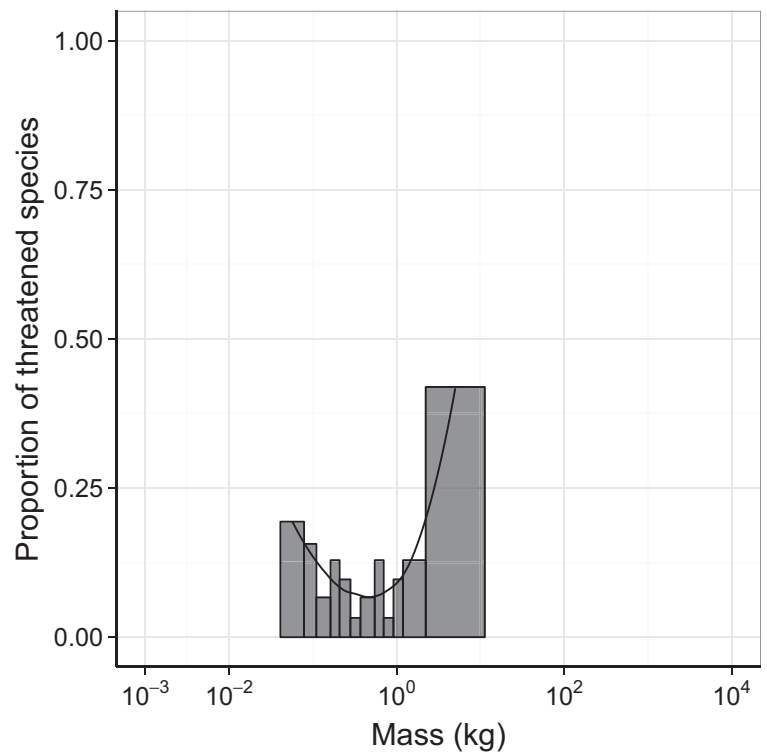

Carnivorous birds

c)

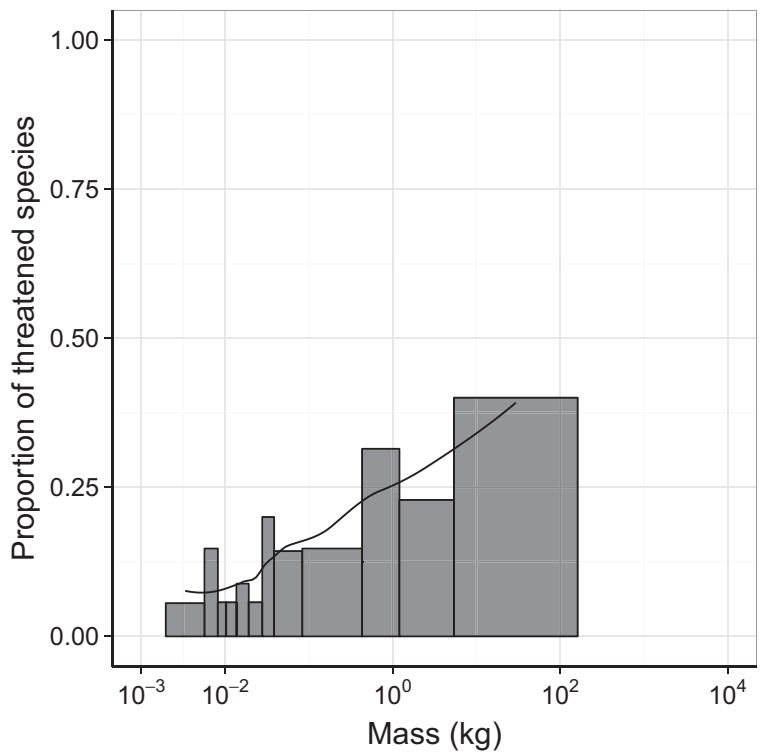

Carnivorous mammals b)

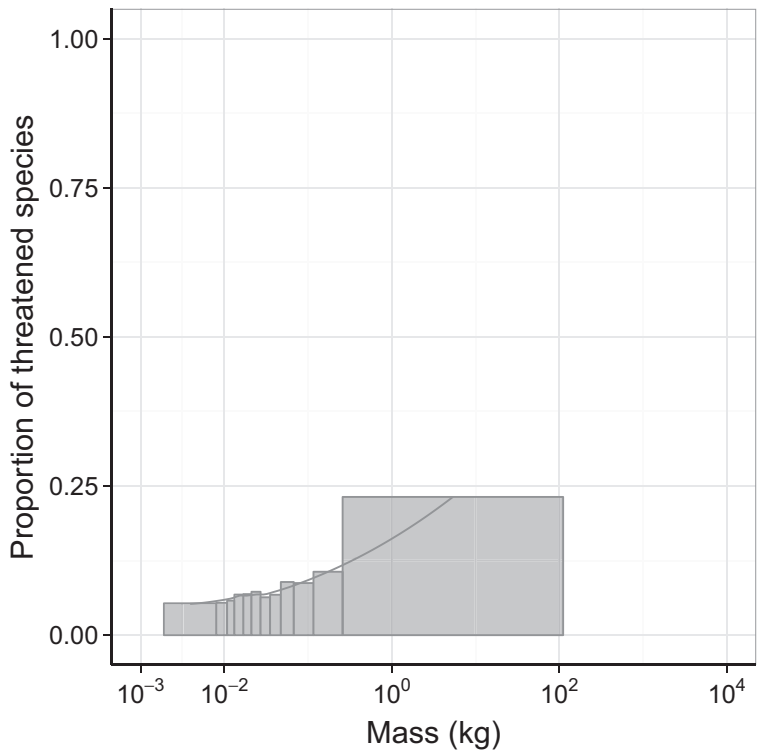

Non-carnivorous birds

d)

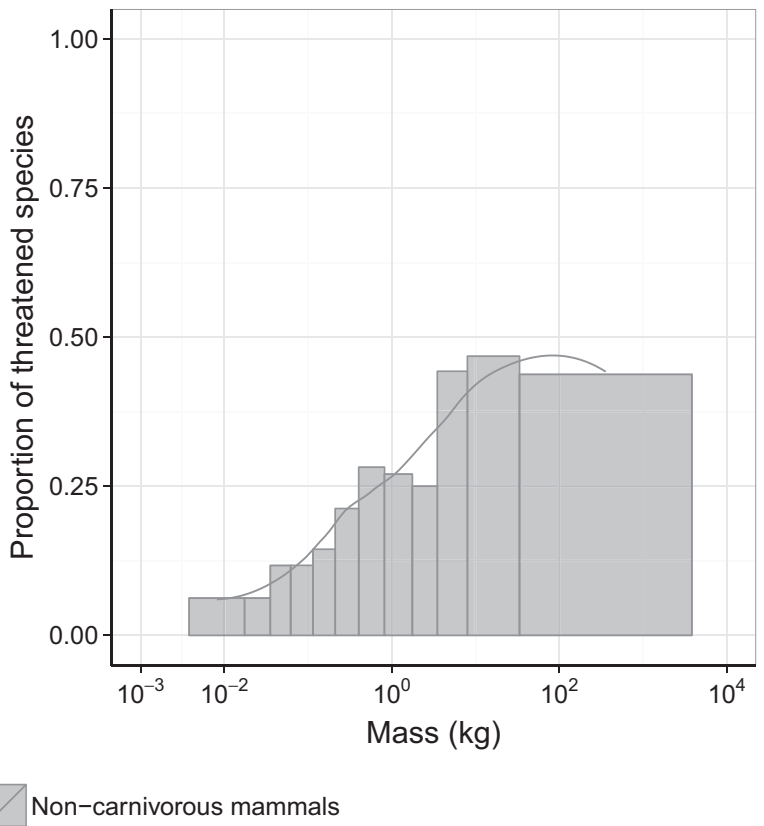

FIG. 5. The proportion of threatened species as listed by IUCN in relation to body size ( $N=12$ bins) for (a) carnivorous birds, (b) non-carnivorous birds, (c) carnivorous mammals, and (d) non-carnivorous mammals.

geographical range sizes are relatively low, as we found probabilities of extinction close to zero, mean times to extinction larger than $10^{20} \mathrm{yr}$, critical patch sizes smaller than $2 \times 10^{6} \mathrm{~km}^{2}$, and ratios of the critical patch size to the geographical range size close to zero. Species with small geographical range size were found to be more at risk, which is supported by findings of Purvis et al.
(2000), Cardillo et al. (2005), and Davidson et al. (2009), who showed that threatened mammal and bird species as listed by the IUCN have much lower current suitable habitat areas and population densities. However, the high PE we found for birds with small geographical range sizes was accompanied by an MTE of at least $10^{10} \mathrm{yr}$. This discrepancy between the two indicators can be 
explained by a difference in extinction threshold (Fujiwara 2007): in the MTE model of Foley (1994), this threshold is set at a population of one individual, whereas the PE model of Ginzburg et al. (1982) is based on a quasiextinction threshold, which we set at 500 individuals to account for the effects of demographic and genetic stochasticity. In general, dividing CPS by $A$ yielded larger values than the $\mathrm{PE}$ predictions, which may be due to the methodology used here to arrive at an extinction indicator from CPS. Our CPS values and relationships between CPS and body size were similar to those found in Pe'Er et al. (2014), who reported relationships between minimum area requirements and body size divided according to taxa and feeding guild based on a literature search covering 216 terrestrial animal species from 80 studies. Furthermore, CPS values differed a factor 0.2-90 $(\alpha=0.1), 0.04-6(\alpha=0.5)$ and $0.03-3(\alpha=0.9)$ from critical patch sizes estimated by multiplying the inverse of the current population density $\left(N_{\mathrm{D}}\right)$ with a minimum viable population size of 200, as in Hendriks et al. (2009). This indicates that the CPS values found in this study are within realistic ranges.

\section{Application}

Given the high and increasing pressure of anthropogenic activities on global biodiversity, there is an urgent need to better predict the vulnerability of species and/ or species groups to extinction (Cardillo et al. 2005). As it is impossible to protect all species from extinction, policy makers and conservationists often prioritize seriously endangered species or areas that enclose many different species or a large number of vulnerable species (Wilson et al. 2011). The decision to select particular species or areas for conservation must be made carefully in light of information on as many species present as possible. Currently, however, extinction vulnerability estimates are lacking for a large number of species (Morais et al. 2013). Extinction vulnerability is typically quantified based on either detailed, complex matrix models or simpler, unstructured PVA models that include a minimum number of variables necessary to adequately assess a population's viability (Foley 2000, Iwasa et al. 2000). However, the major drawback of species-specific population models, even the relatively simple ones, is that they are highly data intensive. For example, reliable vital rate estimates are available for a few species only, illustrated by the Global Population Dynamics Database containing vital rate time series data for approximately 1800 species (Inchausti and Halley 2001), whereas there are over 1.2 million species listed in the Catalogue of Life and The World's Register of Marine Species (Mora et al. 2011). This study provides a coherent analytical framework that quantifies extinction vulnerabilities using limited data. The comparison with the IUCN data indicates that the framework may be applied to obtain first estimates of the relative extinction risk of datadeficient species, so that conservation targets can be better set (Fagan et al. 2001, Trindade-Filho et al. 2012). Further, our framework can be used to assess changes in the extinction vulnerability due to extrinsic anthropogenic factors like habitat alternation, hunting, and climate change, by quantifying the effects of these anthropogenic drivers on current population sizes, carrying capacity and population growth rates.

\section{ACKNOWLEDGMENTS}

This research project was funded by the European Commission under the Seventh Framework Programme on Environment, ENV.2012.6.3-3: DESIRE - DEvelopment of a System of Indicators for a Resource efficient Europe (grant agreement number 308552).

\section{Literature Cited}

Agosta, S. J., and J. Bernardo. 2013. New macroecological insights into functional constraints on mammalian geographical range size. Proceedings of the Royal Society B 280:20130140.

Akçakaya, H. R., and P. Sjögren-Gulve. 2000. Population viability analyses in conservation planning: an overview. Ecological Bulletins 48:9-21.

Anderson, S. C., R. G. Farmer, F. Ferretti, A. L. S. Houde, and J. A. Hutchings. 2011. Correlates of vertebrate extinction risk in Canada. BioScience 61:538-549.

BirdLife International. 2013. Data from IUCN/birdlife species information service, November 2013 release. http://www. birdlife.org. Last assessed on 7 March 2014.

Bowman, J. 2003. Is dispersal distance of birds proportional to territory size? Canadian Journal of Zoology 81:195-202.

Brown, J. H., J. F. Gillooly, A. P. Allen, V. M. Savage, and G. B. West. 2004. Toward a metabolic theory of ecology. Ecology 85:1771-1789.

Buchmann, C. M., F. M. Schurr, R. Nathan, and F. Jeltsch. 2013. Habitat loss and fragmentation affecting mammal and bird communities - the role of interspecific competition and individual space use. Ecological Informatics 14:90-98.

Carbone, C., and J. L. Gittleman. 2002. A common rule for the scaling of carnivore density. Science 295:2273-2276.

Cardillo, M., G. M. Mace, K. E. Jones, J. Bielby, O. R. P. Bininda-Emonds, W. Sechrest, C. D. L. Orme, and A. Purvis. 2005. Multiple causes of high extinction risk in large mammal species. Science 309:1239-1241.

Damuth, J. 1981. Population density and body size in mammals. Nature 290:699-700.

Damuth, J. 1987. Interspecific allometry of population density in mammals and other animals: the independence of body mass and population energy-use. Biological Journal of the Linnean Society 31:193-246.

Davidson, A. D., M. J. Hamilton, A. G. Boyer, J. H. Brown, and G. Ceballos. 2009. Multiple ecological pathways to extinction in mammals. Proceedings of the National Academy of Sciences of the United States of America 106:10702-10705.

Dirzo, R., H. S. Young, M. Galetti, G. Ceballos, N. J. B. Isaac, and B. Collen. 2014. Defaunation in the Anthropocene. Science 345:401-406.

Dunning, J. B. 2007. CRC handbook of avian body masses. Second edition. Taylor \& Francis, Boca Raton, Florida, USA.

Fagan, W. F., E. Meir, J. Prendergast, A. Folarin, and P. Karieva. 2001. Characterizing population vulnerability for 758 species. Ecology Letters 4:132-138.

Foley, P. 1994. Predicting extinction times from environmental stochasticity and carrying capacity. Conservation Biology 8:124-137. 
Foley, P. 2000. Problems in extinction model selection and parameter estimation. Environmental Management 26:S55-S73.

Franklin, I. R. 1980. Evolutionary change in small populations. Pages 135-150 in M. E. Soule and B. A. Wilcox, editors. Conservation biology. An evolutionary-ecological perspective. Sinauer Associates, Sunderland.

Fujiwara, M. 2007. Extinction-effective population index: incorporating life-history variations in population viability analysis. Ecology 88:2345-2353.

Gaston, K. J., and T. M. Blackburn. 1996. Conservation implications of geographic range size body size relationships. Conservation Biology 10:638-646.

Gaston, K., and T. Blackburn. 2008. Pattern and process in macroecology. Blackwell Science, Oxford.

Gillooly, J. F., J. H. Brown, G. B. West, V. M. Savage, and E. L. Charnov. 2001. Effects of size and temperature on metabolic rate. Science 293:2248-2251.

Ginzburg, L. R., L. B. Slobodkin, K. Johnson, and A. G. Bindman. 1982. Quasiextinction probabilities as a measure of impact on population growth. Risk Analysis 2:171-181.

Hakoyama, H., and Y. Iwasa. 2000. Extinction risk of a densitydependent population estimated from a time series of population size. Journal of Theoretical Biology 204:337-359.

Hakoyama, H., and Y. Iwasa. 2005. Extinction risk of a metapopulation: aggregation approach. Journal of Theoretical Biology 232:203-216.

Hendriks, A. J. 2007. The power of size: a meta-analysis reveals consistency of allometric regressions. Ecological Modelling 205:196-208.

Hendriks, A. J., and C. Mulder. 2012. Delayed logistic and Rosenzweig-MacArthur models with allometric parameter setting estimate population cycles at lower trophic levels well Ecological Complexity 9:43-54.

Hendriks, A. J., B. J. C. Willers, H. J. R. Lenders, and R. S. E. W. Leuven. 2009. Towards a coherent allometric framework for individual home ranges, key population patches and geographic ranges. Ecography 32:929-942.

Inchausti, P., and J. Halley. 2001. Investigating long-term ecological variability using the global population dynamics database. Science 293:655-657.

Isaac, N. J. B., D. Storch and C. Carbone. 2011. Taxonomic variation in size-density relationships challenges the notion of energy equivalence. Biology Letters 7:615-618.

IUCN. 2014. The IUCN red list of threatened species. Version 2014.3. http://www.iucnredlist.org. Last assessed on 5 March 2015.

Iwasa, Y., H. Hakoyama, M. Nakamaru, and J. Nakanishi. 2000. Estimate of population extinction risk and its application to ecological risk management. Population Ecology 42:73-80.

Jetz, W., C. Carbone, J. Fulford, and J. H. Brown. 2004. The scaling of animal space use. Science 306:266-268.

Jones, K. E., et al. 2009. PanTHERIA: a species-level database of life history, ecology, and geography of extant and recently extinct mammals. Ecology 90:2648-2648.

Kitzes, J., and A. Merenlender. 2013. Extinction risk and tradeoffs in reserve site selection for species of different body sizes. Conservation Letters 6:341-349.

Lande, R., S. Engen and B.-E. Saether. 2003. Stochastic population dynamics in ecology and conservation. Oxford Series in Ecology and Evolution:224.

Liow, L. H., M. Fortelius, E. Bingham, K. Lintulaakso, H. Mannila, L. Flynn, and N. C. Stenseth. 2008. Higher origination and extinction rates in larger mammals. Proceedings of the National Academy of Sciences of the United States of America 105:6097-6102.

McKinney, M. L. 1997. Extinction vulnerability and selectivity: combining ecological and paleontological views. Annual Review of Ecology and Systematics 28:495-516.

Mora, C., D. P. Tittensor, S. Adl, A. G. B. Simpson, and B. Worm. 2011. How many species are there on earth and in the ocean? PLoS Biology 9:e1001127.

Morais, A. R., M. N. Siqueira, P. Lemes, N. M. Maciel, P. De Marco Jr, and D. Brito. 2013. Unraveling the conservation status of Data Deficient species. Biological Conservation 166:98-102.

Pe'er, G., et al. 2013. A protocol for better design, application, and communication of population viability analyses. Conservation Biology 27:644-656.

Pe'er, G., et al. 2014. Toward better application of minimum area requirements in conservation planning. Biological Conservation 170:92-102.

Pereira, H. M., and G. C. Daily. 2006. Modeling biodiversity dynamics in countryside landscapes. Ecology 87:1877-1885.

Pereira, H. M., G. C. Daily, and J. Roughgarden. 2004. A framework for assessing the relative vulnerability of species to land-use change. Ecological Applications 14:730-742.

Pimm, S. L., H. L. Jones, and J. Diamond. 1988. On the risk of extinction. The American Naturalist 132:757-785.

Purvis, A., J. L. Gittleman, G. Cowlishaw and G. M. Mace. 2000. Predicting extinction risk in declining species. Proceedings of the Royal Society of London. Series B: Biological Sciences 267:1947-1952.

R Core Team. 2014. R: a language and environment for statistical computing. R Foundation for Statistical Computing, Vienna, Austria.

Santini, L., M. Di Marco, P. Visconti, D. Baisero, L. Boitani, and C. Rondinini. 2013. Ecological correlates of dispersal distance in terrestrial mammals. Hystrix 24:181-186.

Savage, V. M., J. F. Gillooly, J. H. Brown, G. B. West, and E. L. Charnov. 2004a. Effects of body size and temperature on population growth. American Naturalist 163:429-441.

Savage, V. M., J. F. Gillooly, W. H. Woodruff, G. B. West, A. P. Allen, B. J. Enquist, and J. H. Brown. 2004b. The predominance of quarter-power scaling in biology. Functional Ecology 18:257-282.

Sinclair, A. R. E. 2003. Mammal population regulation, keystone processes and ecosystem dynamics. Philosophical Transactions of the Royal Society of London. Series B: Biological Sciences 358:1729-1740.

Skellam, J. G. 1951. Random dispersal in theoretical populations. Biometrika 38:196-218.

Smith, F. A., and S. K. Lyons. 2013. The way forward. Pages 235-246 in F. A. Smith and S. K. Lyons, editors. Animal body size: linking pattern and process across space, time and taxonomy. University of Chicago Press, Chicago.

Trindade-Filho, J., R. de Carvalho, D. Brito, and R. Loyola. 2012. How does the inclusion of Data Deficient species change conservation priorities for amphibians in the Atlantic Forest? Biodiversity \& Conservation 21:2709-2718.

West, G. B., and J. H. Brown. 2005. The origin of allometric scaling laws in biology from genomes to ecosystems: towards a quantitative unifying theory of biological structure and organization. Journal of Experimental Biology 208:1575-1592.

Wilson, K. A., M. C. Evans, M. Di Marco, D. C. Green, L. Boitani, H. P. Possingham, F. Chiozza, and C. Rondinini. 2011. Prioritizing conservation investments for mammal species globally. Philosophical Transactions of the Royal Society B: Biological Sciences 366:2670-2680.

\section{SUPPORTING INFORMATION}

Additional supporting information may be found in the online version of this article at http://onlinelibrary.wiley.com/ doi/10.1890/14-2019.1/suppinfo 